\title{
Efficient Multi-method Rule Learning for Pattern Classification Machine Learning and Data Mining
}

\author{
Chinmay Maiti ${ }^{1}$ and Somnath $\mathrm{Pal}^{2}$ \\ ${ }^{1}$ Dept. of Info. Tech. \\ Jadavpur University \\ chinmay@it.jusl.ac.in \\ ${ }^{2}$ Dept. of Comp. Sc. \& Tech. \\ Bengal Engg. \& Sc. University \\ sp@cs.becs.ac.in
}

\begin{abstract}
The work presented here focuses on combining multiple classifiers to form single classifier for pattern classification, machine learning for expert system, and data mining tasks. The basis of the combination is that efficient concept learning is possible in many cases when the concepts learned from different approaches are combined to a more efficient concept. The experimental result of the algorithm, EMRL in a representative collection of different domain shows that it performs significantly better than the several state-of-the-art individual classifier, in case of 11 domains out of 25 data sets whereas the state-of-the-art individual classifier performs significantly better than EMRL only in 5 cases.
\end{abstract}

Keywords: Machine learning, Multiple Classifiers, Missing values, Discretization, Classification.

\section{Introduction}

The task of constructing ensembles of classifiers [7 can be categorized into subtasks. One approach is to generate classifiers by applying different learning algorithm with heterogeneous 12 model representations to a single data set.As per our knowledge, such approach was introduced for decision tree learning in 15 and was subsequently improved in [16]. Another approach is to apply a single learning algorithm with different parameters settings to a single data set. Then, methods like bagging 2 and boosting 9 generate multiple classifiers by applying a single learning algorithm to different versions of a given data set. Bagging produces replicate training sets by sampling with replacement from the training instances. Boosting considers all instances at each repetition, but maintains a weight for every instance in the training set that reflects its importance, re-weighting of the misclassified instances leads to different classifiers. In both cases, voting to form a composite classifier combines the multiple classifiers. In bagging, each component classifier has the equal vote, whereas in boosting uses different voting strengths to each component classifiers on the basis of their accuracy. 
This paper here focuses on the combining multiple classifiers using multimethod approach for pattern classification, machine learning for expert system and data mining task. This method is to apply different learning algorithms with homogeneous model representation on a single data set and the combinational algorithm is called EMRL(Efficient Multi-method Rule Learning). The algorithm EMRL heuristically combines the outputs of different state-of-the-art rule inducing algorithms, namely C4.5rules [18, CN2 [5] 6],RISE 8, and PRISM 4 and produces efficient composite classifier. The following section briefly describes EMRL algorithm. The experimental evaluation of EMRL algorithm for comparison of performances with the state-of-the-art individual classifier is described in section 3. Finally, the conclusion is summarized in the last section.

\section{EMRL Algorithm}

\subsection{The Basis of EMRL Algorithm}

Fundamentally, EMRL(Efficient Multi-method Rule Learning) provides a framework to combine the outputs of various base classifiers of empirical inductive generalization, namely C4.5rules, CN2, RISE and PRISM. This is done by heuristically combining outputs of different classifiers in the rule plane. This approach is called as multi-method learning in [15] for decision tree plane.

The heuristic that is the basis of this paper is that efficient concept learning is possible in many cases when the concepts learned from different approaches are combined to a more efficient concept. EMRL algorithm performs in two steps. It generates sets of rules for a given instance set by using different rule inducing algorithms, and then combines the rule set to get an efficient composite classifier.

\subsection{The EMRL Algorithm}

The Efficient Multimethod Rule Learning(EMRL) algorithm is given below. The objective of EMRL algorithm is to select a resultant set of rules which is able enough to classify the example set completely and successfully. An EMRL algorithm is based on k-fold cross-validation of the training data sets to select the best possible set of rules generated by $\mathrm{k}$ numbers of (k-1) parts forming temporary training sets with $\mathrm{k}$ numbers of one part formimg temporary test set. It then generates the set of rulesets obtained from $m(m \geq 2)$ rule inducing algorithm like C4.5rules, CN2, RISE, PRISM for each temporary training set. Next, it computes the union of the rulesets and then calculates the weighted vote for each rule using the corresponding temporary training data set. First, the weighted vote is initialized to zero for each rule in the unified set. Then weighted vote is computed for each rule one by one. The weighted vote of a rule $=$ the number of instances in the temporary training set correctly classified by the rule. Next, the weighted vote for each rule is modified using the instances in the temporary test set.

Modification of vote for each rule is done whether the rule classifies an instance correctly or misclassifies. If an instance is classified correctly by the rule 
then weighted vote of that rule is increased by adding RV(reward vote) to the existing vote. Again, if an instance is misclassified by it then decrease the vote by subtracting PV(penalty vote)from the existing vote. If an instance is not covered by the rule 1 then no changes to the vote.In EMRL algorithm both reward and penalty vote are totally experimental. After modification of weighted vote for rules in all rule sets $R_{j} j=1, \ldots, m(m \geq 2)$ compute the average weight and the final average weighted vote is determined by using the following formula Final Average Weighted Vote $=($ average weighted vote $\times$ APW $) / 100$.

The APW(Acceptable Percentage of Weight)value is also experimental and varying from 1 to 100 (in steps of $\epsilon$ ). Here, APW acts as a factor for varying the threshold value of avreage weighted vote which is used for final selection of rules in the accetable rule set. Now, the computed final weighted vote is used to select the best possible set of rules. The rule with weighted vote greater than or equal to the final weighted vote is considered in the final set.

\section{EMRL Algorithm}

Step1. Divide the training set into n mutually exclusive parts and prepare temporary training data set $\left(L_{i}\right)$ with $(\mathrm{n}-1)$ parts and call the rest one part as temporary test data set $\left(T_{i}\right)$. Generate $L_{i}$ and $T_{i}, i=1,2, \ldots, n$.

Step2. For each $L_{i}$ generate Rule sets $R_{j}, j=1, \ldots, m(m \geq 2)$ by applying $m$ rule including algorithms.

Step3. Construct union of all input rule sets $R_{j}, j=1, \ldots, m$.

Step4. For all rules initialize weighted vote as zero.

Step5. Calculate the weighted vote for each rule using temporary training data set $L_{i}$, i.e., weighed vote of a rule is equal to the Number of instances correctly classified by it.

Step6. Modify the weighted vote for each rule of the union set using temporary test data set $T_{i}$

a) If an instance is correctly classified by a rule then increase the weighted vote of that rule by RV amount (user defined reward vote).

b) If an instance is not covered by a rule then do nothing to the weighted vote of that rule.

c) If an instance is misclassified by a rule then decrease the weighted-vote of that rule by PV amount (user defined penalty vote).

Step7. Calculate the average weighted vote and modify it by the formula

Final average weighted vote $=($ average weighted vote $\times$ APW $) / 100($ APW means Acceptable Percentage of Weight varying from 1 to 100).

Step8. Composite efficient rule set is then formed by selecting the rules from union of all $R_{j}$ 's and having weighted vote greater than or equal to the modified weighted vote.

\footnotetext{
${ }^{1}$ A rule in a rule set may classify or misclassify an instance or may not be applicable to the instance at all. The rule that is not applicable to an instance is considered as not covering the instance.
} 


\subsection{Time Complexity}

Worst case time complexity of the algorithm EMRL is $\mathrm{O}\left(m r^{2}\right)+\mathrm{O}($ mer), where $\mathrm{m}$ is the number of terms in each rule, $\mathrm{e}$ is the total number of instances in the training set and $\mathrm{r}$ is the number of unified rules.

\section{Empirical Evaluation}

An empirical evaluation is carried out with the goal of testing whether at all improvement is possible in the multi-method framework proposed in EMRL algorithm.

\subsection{Data Sets}

Empirical calculations of the performance of EMRL algorithm are carried out on 25 real world data sets drawn from the University of California at Irvin data repository [14. The data sets are chosen vary across a number of dimensions including the type of application area, the type of features in the data sets, the number of output classes and the number of instances. These are data sets with both discrete and continuous data.

\subsection{Treatment of Missing Values}

Real-world data is often incomplete and often has missing values. Several authors 13 18] have developed different answers to this problem, usually based either on filling in missing attributes values with most probable value or a value determined by exploiting interrelation-ship among the values of different attributes, or on looking at the probability distribution of values of attributes. In this work 17, a different approach is followed in the treatment of missing values. The missing values in attribute means that a particular test is not performed (and hence not required) to classify the particular instance. This itself is information. To keep this information in resulting rules the missing values of an attribute are assigned a separate value is chosen as the highest value of the attribute which would not be the case with other methods. Such treatment of missing values as a separate value is well-known in machine learning literature [10.

\subsection{Discretization}

Efficient discretization of continuous attributes is an important because it has effects on speed, accuracy and understandability of the induction models. Furthermore, many machine learning alogorithms such as PRISM [4 can not handle continuous features. All data sets are discretized using global discretization based on successive pseudo deletion of instances [17] to reduce the conflicting instances, i.e., by successive reduction of noises in the database. Pre-discretization of data is also a step towards learning itself and can be considered as an elementary inductive method that predicts the local majority class in each learned interval 24] and therefore, adds informations unlike loss of information to the model. 


\subsection{Experimental Design}

We have considered C4.5rules, CN2, RISE and PRISM rule inducing algorithms as base learners for EMRL algorithm. The EMRL, being a general framework, can combine output rules of any two or more base rule inducing algorithms. All possible combinations of C4.5rules, CN2, RISE and PRISM have tried using EMRL for all 25 data sets. In our experimental design, we set reward vote as one and penalty vote as two. There is a basis that why the penalty vote is greater than the reward vote. The task of EMRL algorithm is to find the best possible set of rules which are efficient in future for prediction. So, EMRL is trying to severely penalize the rules which are less significant to classify the instances and penalize them by decreasing the vote by twice the amount than for successful classification when it misclassifies an instance. Althoug we have conducted our experiment with $R V=1$ and $P V=1$, but the chosen values with $R V=1$ and $P V=2$ were formed to result in slightly improved accuracy. Also, in our experimental design to determine the value of APW, we make several runs of the EMRL algorithm with interval $5(\epsilon=5)$ of the APW value starting from 20 to 70 (instead of 1 to 100 because the performance of the final acceptable rule set degrades drastically beyond APW value of 20 and 70) and finally set the value as 45 where it provides maximum accuracy for most data sets. We have considered the combination with highest predictive accuracy and compare with the best of single state-of-the-art base algorithms and that is reported here. The accuracies have calculated using k-fold cross-validation method. Here, we set the value of $\mathrm{k}$ equals to 10 . Such 10-fold cross-validation experiments are repeated 5 times for each of the data sets and each of the possible combination of algorithms. Thus, accuracy calculations are averaged over 50 values for each data set and each combination.

\subsection{Experimental Results}

The Table 1 shows empirical results in terms of average accuracies for both EMRL ( combination with highest accuracies) and best of single state-of-theart base algorithms. Superscripts indicates confidence level for difference between the EMRL and state-of-the-art base algorithm using one tailed paired t-test. The results of Table 1 are interesting in themselves; however, they are summarized in a few global measures of comparative performance in Table 2.

\section{Related Work}

The main idea of ensemble methodology is to combine a set of models, each of which solves the same original task, in order to obtain a better composite global model, with more accurate and reliable estimates or decisions than can be obtained from using a single model.The strategy of combining the classifiers generated by an induction algorithm 19. The simplest combiner determines the output solely from the outputs of the individual inducers. Ali and Pazzani [1] 
Table 1. Empirical Result: acc.=average accuracy and s.d.=standard deviation. Superscripts denote confidence level : 1 is $99.5 \%, 2$ is $99 \%, 3$ is $97.5 \%, 4$ is $95 \%, 5$ is $90 \%$, and 6 is below 90\%. Symbols denote the algorithms : A1 is C4.5rules, A2 is CN2, A3 is RISE, A4 is PRISM.

\begin{tabular}{|l|c|c|c|c|}
\hline \multirow{2}{*}{ Data Sets } & \multicolumn{2}{|c|}{ Individual Classifiers } & \multicolumn{2}{c|}{ EMRL } \\
\cline { 2 - 5 } & $a c c . \pm s . d$. & Algorithm & $a c c . \pm s . d$. & Algorithms Combined \\
\hline heart hungary & $76.39 \pm 8.32$ & $\mathrm{~A} 4$ & $67.02 \pm 9.45^{1}$ & $\mathrm{~A} 3, \mathrm{~A} 4$ \\
\hline heart switzerland & $45.46 \pm 10.56$ & $\mathrm{~A} 2$ & $46.47 \pm 10.47^{6}$ & $\mathrm{~A} 1, \mathrm{~A} 3, \mathrm{~A} 4$ \\
\hline heart cleveland & $58.86 \pm 7.28$ & $\mathrm{~A} 2$ & $61.42 \pm 7.51^{3}$ & $\mathrm{~A} 3, \mathrm{~A} 4$ \\
\hline primary-tumar & $42.52 \pm 7.15$ & $\mathrm{~A} 2$ & $48.42 \pm 8.44^{1}$ & $\mathrm{~A} 1, \mathrm{~A} 2$ \\
\hline iris & $96.65 \pm 3.35$ & $\mathrm{~A} 1$ & $95.33 \pm 3.06^{2}$ & $\mathrm{~A} 1, \mathrm{~A} 2 / \mathrm{A} 2, \mathrm{~A} 3$ \\
\hline glass & $72.86 \pm 7.36$ & $\mathrm{~A} 3$ & $75.19 \pm 7.41^{4}$ & $\mathrm{~A} 1, \mathrm{~A} 2, \mathrm{~A} 3, \mathrm{~A} 4$ \\
\hline pima-indians & $74.47 \pm 6.02$ & $\mathrm{~A} 1$ & $76.56 \pm 5.92^{3}$ & $\mathrm{~A} 1, \mathrm{~A} 2$ \\
\hline liver disorder & $67.30 \pm 7.22$ & $\mathrm{~A} 3$ & $71.64 \pm 6.96^{1}$ & $\mathrm{~A} 2, \mathrm{~A} 3$ \\
\hline hepatitis & $85.29 \pm 8.06$ & $\mathrm{~A} 3$ & $85.87 \pm 6.28^{6}$ & $\mathrm{~A} 2, \mathrm{~A} 3$ \\
\hline cpu-performance & $59.14 \pm 14.99$ & $\mathrm{~A} 3$ & $58.24 \pm 13.96^{6}$ & $\mathrm{~A} 1, \mathrm{~A} 2, \mathrm{~A} 3, \mathrm{~A} 4$ \\
\hline dermatology & $94.85 \pm 5.60$ & $\mathrm{~A} 3$ & $80.30 \pm 10.78^{1}$ & $\mathrm{~A} 2, \mathrm{~A} 3$ \\
\hline hdva & $31.50 \pm 13.24$ & $\mathrm{~A} 4$ & $34.00 \pm 8.31^{5}$ & $\mathrm{~A} 2, \mathrm{~A} 4$ \\
\hline credit & $86.09 \pm 4.31$ & $\mathrm{~A} 3$ & $87.68 \pm 3.12^{2}$ & $\mathrm{~A} 1, \mathrm{~A} 2, \mathrm{~A} 3$ \\
\hline ecoli & $81.26 \pm 4.71$ & $\mathrm{~A} 3$ & $82.74 \pm 6.45^{4}$ & $\mathrm{~A} 2, \mathrm{~A} 3$ \\
\hline hypothyroid & $99.20 \pm 0.39$ & $\mathrm{~A} 1$ & $99.30 \pm 0.31^{4}$ & $\mathrm{~A} 1, \mathrm{~A} 2, \mathrm{~A} 3$ \\
\hline wisconsin & $95.29 \pm 2.57$ & $\mathrm{~A} 2$ & $97.28 \pm 1.35^{1}$ & $\mathrm{~A} 1, \mathrm{~A} 2$ \\
\hline wine & $98.33 \pm 3.56$ & $\mathrm{~A} 3$ & $98.33 \pm 3.56^{6}$ & $\mathrm{~A} 3, \mathrm{~A} 4$ \\
\hline echo cardiogram & $65.99 \pm 11.69$ & $\mathrm{~A} 3$ & $66.65 \pm 8.55^{6}$ & $\mathrm{~A} 1, \mathrm{~A} 3$ \\
\hline breast cancer & $72.71 \pm 7.29$ & $\mathrm{~A} 3$ & $74.50 \pm 6.04^{4}$ & $\mathrm{~A} 2, \mathrm{~A} 3$ \\
\hline voting & $95.39 \pm 3.09$ & $\mathrm{~A} 1$ & $96.10 \pm 3.67^{5}$ & $\mathrm{~A} 2, \mathrm{~A} 3$ \\
\hline zoo & $97.00 \pm 6.40$ & $\mathrm{~A} 2$ & $94.00 \pm 6.63^{2}$ & $\mathrm{~A} 1, \mathrm{~A} 2, \mathrm{~A} 3 / \mathrm{A} 2, \mathrm{~A} 3, \mathrm{~A} 4$ \\
\hline lymphography & $82.34 \pm 9.30$ & $\mathrm{~A} 2$ & $85.10 \pm 11.14^{4}$ & $\mathrm{~A} 2, \mathrm{~A} 3$ \\
\hline new-thyroid & $99.09 \pm 2.73$ & $\mathrm{~A} 2 / \mathrm{A} 3$ & $99.09 \pm 2.73^{6}$ & $\mathrm{~A} 2, \mathrm{~A} 3$ \\
\hline annealing & $99.50 \pm 0.83$ & $\mathrm{~A} 3$ & $98.75 \pm 0.97^{1}$ & $\mathrm{~A} 1, \mathrm{~A} 2$ \\
\hline imports-85 & $79.62 \pm 8.44$ & $\mathrm{~A} 2$ & $78.67 \pm 9.54^{6}$ & $\mathrm{~A} 1, \mathrm{~A} 2, \mathrm{~A} 4$ \\
\hline
\end{tabular}

Table 2. Summary of The Results

\begin{tabular}{|c|c|c|}
\hline $\begin{array}{c}\text { Algorithm } \rightarrow \\
\text { Measure } \downarrow\end{array}$ & $\begin{array}{c}\text { State-of-the-art } \\
\text { Individual Classifier }\end{array}$ & (C4.5rules,CN2,RISE,PRISM) \\
\hline $\begin{array}{c}\text { Average Accuracy } \\
\text { Over 25 data sets }\end{array}$ & 77.92 & 78.34 \\
\hline $\begin{array}{c}\text { Number of wins in } \\
\text { Accuracy }\end{array}$ & 7 & 16 \\
\hline $\begin{array}{c}\text { Number of significant } \\
\text { wins in Accuracy }\end{array}$ & 5 & 11 \\
\hline $\begin{array}{c}\text { Wilcoxson sign rank } \\
\text { test in Accuracy }\end{array}$ & -- & $90.56 \%$ \\
\hline
\end{tabular}


have compared several combination methods: uniform voting, Bayesian combination, distribution summation and likelihood combination. Moreover, theoretical analysis have been developed for estimating classification improvement [21. Along with simple combiners there are other more sophisticated method, such as stacking [23]. On the otherhand, there is the success of bagging [2] and boosting 9] and similar other re-sampling techniques. These have the disadvantage of generating incomprehensible concept descriptions. MDTL2 [15], MDTL3.1 [16] was a valuable tool for combining large rule sets into simpler comprehensible decision tree. EMRL algorithm presented here combines rulesets generated by different heterogeneous classifiers into a single efficient set of rules. The difference of MDTL and EMRL type of combination of classifiers with other ensemble and multiple classifier systems is that the former type use the concept description of an ensemble of classifiers (instead of their output) for combination whereas the latter type use the outputs of emsemble of classifiers for the classification tasks. Although, the resultant classifier from MDTL and EMRL type of algorithm can be used for bagging, boosting, voting or any other classifier combination method like Data Envelop Analysis Weighting method [20], logarithmic Opinion Pool 11, Order Statistics 22, to produce still improved results.

\section{Conclusion and Future Research}

Recently, combining classifiers to achieve greater accuracy has become an increasingly important research topic in machine learning. In this paper, we describes an algorithm EMRL that can combine various base rule inducing algorithm into an efficient composite classifier. The empirical evaluation of the algorithm shows that combination of state-of-the-art base classifier using EMRL produced significantly better accuracies than best of single state-of-the-art base classifier. Further, performance improvement can possibly be expected if EMRL is incorporated as a base classifier in different ensemble methods such as bagging, boosting, voting(heterogeneous) etc methods on which our current work is going on. Other direction of future research include applicability of EMRL to large databases.

\section{References}

1. Ali, K.M., Pazzani, M.J.: Error Reduction through Learning Multiple Descriptions. Machine Learning 24(3), 173-202 (1996)

2. Breiman, L.: Bagging Predictors. Machine Learning 24(2), 123-140 (1996)

3. Bruzzon, L., Cossu, R.: A Robust Multiple Classifier System for a Partially Unsupervised Updating of Land-cover Maps. In: Kittler, J., Roli, F. (eds.) MCS 2001. LNCS, vol. 2096, pp. 259-268. Springer, Heidelberg (2001)

4. Candrowska, J.: PRISM: An algorithm for inducing modular rules. International Journal for Man-Machine Studies 27, 349-370 (1987)

5. Clark, P., Boswell, R.: Rule Induction with CN2: Some recent improvements. In: Proc. of the $5^{\text {th }}$ ECML, Berlin, pp. 151-163 (1991) 
6. Clark, P., Niblett, T.: The CN2 Induction Algorithm. ML 3, 261-283 (1989)

7. Dietterich, T.G.: Machine Learning Research: Four Current Directions. AI Magazine 18(4), 97-136 (1997)

8. Domings, P.: Unification Instance-based and Rule-base Induction. Machine Learning 3, 139-168 (1996)

9. Freund, Y., Schapire, R.E.: Experiments with a New Boosting Algorithm. In: Proc. of the $13^{\text {th }}$ International Conference on Machine Learning, pp. 146-148. Morgan Kaufmann, San Francisco (1996)

10. Grzymala-Busse, J.W., Grzymala-Busse, W.J.: Handling Missing Attributes Values. In: Maimon, O., Rokach, L. (eds.) Data Mining \& Knowledge Discovery Hand Book, pp. 35-57. Springer, Heidelberg (2005)

11. Hansen, J.: Combining Predictors Meta Machine Learning Methods abd Bias Variance \& Ambiguity Decompositions Ph. D. dissertation, Aurhns University (2000)

12. Merz, C.J.: Using Correspondence Analysis to Combine Classifiers. Machine Learning 36(1/2), 33-58 (1999)

13. Mingers, J.: An Empirical Comparison of Selection Measure for Decision-tree Induction. Machine Learning 3, 319-342 (1989)

14. Murphy, P. M.: UCI Repository of Machine Learning Databases (Machine-readable data repository). Department of Information and Computer Science, Irvine, CA: University of California (1999)

15. Pal, S., Saha, T.K., Pal, G.K., Maiti, C., Ghosh, A.M.: Multi-method Decision Tree Learning for Data Mining. In: IEEE ACE 2002, pp. 238-242 (2002)

16. Pal, S., Maiti, C., Mazumdar, A.G., Ghosh, A.M.: Multi-Method Combination For Learning Search-Efficient And Accurate Decision Tree. In: 5-th Intenational conference on Advances in Pattern Recognition, ISI, Kolkata, pp. 141-147 (2003)

17. Pal, S., Maiti, C., Debnath, K., Ghosh, A.M.: SPID3: Discretization Using Pseudo Deletion. Journal of The Institute of Engineers 87, 25-31 (2006)

18. Quinlan, J.R.: Program for Machine Learning. Morgan Kaufmann, CA (1993)

19. Rokach, L.: Ensemble Methods for Classifiers. In: Maimon, O., Rokach, L. (eds.) The Data Mining and Knowledge Discovery Hand Book, pp. 956-980. Springer, Heidelberg (2005)

20. Sohn, S.Y., Choi, H.: Ensemble based on Data Envelopment Analysis. In: ECML Meta Learning Workshop (September 2004)

21. Tumer, K., Ghosh, J.: Linear and Order Statistics Combiners for Pattern Classification. In: Sharkey, A. (ed.) Combining Artificial Neural Nets, pp. 127-162. Springer, Heidelberg (1999)

22. Tumer, K., Ghosh, J.: Robust Order Statistics based Ensemble for Distributed Data Mining. In: Kargupto, H., Chan, P. (eds.) Advances in Distributed and Parallel Knowledge Discovery, pp. 185-210. AAAI / MIT Pess (2000)

23. Wolpert, D.: Stacked Generalization. Neural Networks 5(2), 241-260 (1992)

24. Zighed, D.A., Rabaseda, S., Rakotomalala, R., Feschet, F.: Discretization methods in supervised learning. In: Ency. of Comp. Sc.\& Tech. vol. 44, pp. 35-50. Marcel Dekker Inc (1999) 